\title{
Comprendre la construction sociale de la
} représentativité syndicale

Un éclairage à partir des branches professionnelles

Analysing the Social Construction of Trade Unions' Representativeness in French Sectorial Branches

\section{Tristan Haute et Karel Yon}

\section{OpenEdition}

\section{Journals}

Édition électronique

URL : http://journals.openedition.org/travailemploi/8282

DOI : $10.4000 /$ travailemploi.8282

ISSN : 1775-416X

\section{Éditeur}

DARES - Ministère du Travail

\section{Édition imprimée}

Date de publication : 1 avril 2018

Pagination : 101-124

ISSN : 0224-4365

\section{Référence électronique}

Tristan Haute et Karel Yon, "Comprendre la construction sociale de la représentativité syndicale », Travail et Emploi [En ligne], 154 | avril-juin 2018, mis en ligne le 11 juillet 2019, consulté le 05 septembre 2019. URL : http://journals.openedition.org/travailemploi/8282 ; DOI : 10.4000/travailemploi.8282 


\title{
Comprendre la construction sociale de la représentativité syndicale
}

\section{Un éclairage à partir des branches professionnelles}

\author{
Tristan Haute ${ }^{*}$ Karel Yon ${ }^{* *}$
}

\begin{abstract}
Depuis l'institution de la «Mesure d'audience de la représentativité syndicale » (Mars) en 2008, les suffrages des salariés conditionnent la représentativité légale des syndicats et la validité des accords collectifs. Les résultats de la représentativité syndicale et leur construction, sur le plan national interprofessionnel, ont été beaucoup commentés, la victoire de la CFDT sur la CGT étant considérée comme un bouleversement profond du champ syndical. On s'est peu intéressé à la situation dans les branches professionnelles. Or une analyse à cette échelle apporte un éclairage original. D'une part, elle souligne la rupture entre le nouveau dispositif de quantification et les pratiques antérieures de recueil et d'analyse des données des élections professionnelles. C'est en effet au niveau des branches que la spécificité des résultats issus de Mars, qui agrège des données électorales hétérogènes, est la plus visible. D'autre part, l'analyse donne à voir un paysage syndical plus complexe que celui qui ressort de la mesure d'audience interprofessionnelle. Le pluralisme est bien plus riche dans les branches, même si les résultats confirment la suprématie du couple CGT-CFDT, sans que la CFDT ne se détache véritablement de sa rivale.
\end{abstract}

T e 31 mars 2017, sont publiés les résultats fondant la représentativité des organisations syndicales de salariés au niveau national interprofessionnel. À cette occasion, la Confédération française démocratique du travail (CFDT) devance pour la première fois la Confédération générale du travail (CGT) dans le secteur privé, conduisant certains commentateurs à décrire cet événement comme un « séisme »

\footnotetext{
* Univ. Lille, CNRS, UMR 8026 - CERAPS - Centre d'études et de recherches administratives politiques et sociales, F-59000 Lille, France ; tristan.haute@ orange.fr.

** Univ. Lille, CNRS, UMR 8026 - CERAPS - Centre d'études et de recherches administratives politiques et sociales, F-59000 Lille, France ; ynkarel@yahoo.fr.
} 
historique dans le paysage syndical français ${ }^{1}$. Considérées comme un «baromètre des audiences syndicales et des changements intervenus [...] sur la scène sociale française » (LABBÉ, 1994, p. 7), les élections professionnelles ont acquis un rôle décisif depuis la loi de 2008 «portant rénovation de la démocratie sociale ». L'audience syndicale conditionne en effet désormais la représentativité légale des syndicats et la validité des accords collectifs (BÉROUD et al., 2012). Elle est déterminée à partir des résultats produits par Mars (pour Mesure d'audience de la représentativité syndicale), dispositif national de recueil et d'agrégation de données électorales syndicales. Puis elle est validée par les organisations syndicales et patronales représentatives, réunies avec l'administration du travail au sein du Haut Conseil du dialogue social' ${ }^{2}$. Avec Mars, les chercheurs disposent d'un matériau empirique nouveau pour étudier les relations professionnelles et le syndicalisme ${ }^{3}$. Cette base permet une observation du paysage syndical et de ses évolutions avec actuellement des données disponibles pour deux cycles électoraux (2009-2012 et 2013-2016).

À ce jour, peu de travaux ont analysé les élections professionnelles selon la nouvelle donne, post-2008, si ce n'est à travers un prisme qualitatif (LE Digol, VoILliot, 2011 ; BÉROUd, Yon, 2014 ; HEGE et al., 2015). Certains chercheurs ont focalisé leur attention sur la représentativité nationale interprofessionnelle, calculée à partir d'un corps électoral d'un peu plus de 13 millions de salariés (tableau 1), et sur le dispositif de mesure lui-même (ANDOLFATTO, 2018). Les résultats au niveau des branches professionnelles ont été largement moins investigués et lesdites branches sont en général utilisées comme une simple échelle pour la désagrégation des données électorales (HAUTE, 2016, 2018). Dans cet article, nous voudrions montrer l'intérêt de considérer cet échelon d'analyse en tant que tel. C'est en effet à l'échelle conventionnelle ${ }^{4}$ que s'observent le plus nettement les changements induits par la réforme des règles de représentativité syndicale.

Au premier abord, la branche professionnelle pourrait sembler une institution dépassée. La principale tendance dans le domaine des relations professionnelles est en effet à la décentralisation de la négociation collective, faisant de l'entreprise le principal cadre de production normative. Cependant, bien que la réforme de 2008 participe de ce mouvement en déplaçant au niveau des établissements la source de la représentativité syndicale, elle a aussi contribué à conforter le mouvement déjà noté d'institutionnalisation des branches professionnelles (TALLARD, 2004). Elle dissocie en effet les branches des acteurs qui les font vivre, en substituant le principe de reconnaissance par l'audience électorale à l'ancienne logique qui combinait la

1. Michel Noblecourt, « Syndicalisme : la CFDT détrône la CGT de la première place », Le Monde, 31 mars 2017.

2. Un processus de réforme analogue a été engagé dans la fonction publique avec les « accords de Bercy » du 2 juin 2008, transcrits dans la loi du 5 juillet 2010. Nous n'en dirons cependant pas plus dans cet article, car les résultats des élections professionnelles organisées dans les trois fonctions publiques ne sont pas intégrés au dispositif Mars.

3. Les données compilées dans la base Mars nous ont été transmises par plusieurs organisations syndicales que nous tenons à remercier ici.

4. Dans cet article, nous entendons « conventionnel » comme synonyme de « professionnel », au sens de se rapportant aux conventions collectives de branche. 
TABLEAU 1 - Évolution de la mesure d'audience dans le secteur privé

\begin{tabular}{|c|c|c|c|c|}
\hline & \multicolumn{2}{|c|}{ Mars 2013} & \multicolumn{2}{|c|}{ Mars 2017} \\
\hline Inscrits & \multicolumn{2}{|c|}{12755317} & \multicolumn{2}{|c|}{13244736} \\
\hline Votants & \multicolumn{2}{|c|}{5456527} & \multicolumn{2}{|c|}{5664031} \\
\hline Taux de participation & \multicolumn{2}{|c|}{$42,78 \%$} & \multicolumn{2}{|c|}{$42,76 \%$} \\
\hline \multirow[t]{2}{*}{ Exprimés } & \multicolumn{2}{|c|}{5064920} & \multicolumn{2}{|c|}{5243128} \\
\hline & $\begin{array}{c}\text { Nombre de voix } \\
\text { obtenues en } \\
\text { mars } 2013^{* * *}\end{array}$ & $\%$ de voix & $\begin{array}{l}\text { Nombre de voix } \\
\text { obtenues en } \\
\text { mars } 2017^{* * *}\end{array}$ & $\%$ de voix \\
\hline CGT & 1355927,54 & 26,9 & 1302775,50 & 24,9 \\
\hline CFDT & 1317111,84 & 26,1 & 1382646,70 & 26,4 \\
\hline $\mathrm{FO}^{*}$ & 807434,60 & 16,0 & 817570,38 & 15,6 \\
\hline CFE-CGC ${ }^{*}$ & 477459,50 & 9,5 & 559304,51 & 10,7 \\
\hline CFTC $^{*}$ & 470824,51 & 9,3 & 497361,18 & 9,5 \\
\hline Unsa* & 215696,14 & 4,3 & 280554,56 & 5,4 \\
\hline Solidaires & 175557,67 & 3,5 & 181405,44 & 3,5 \\
\hline Autres & 223513,69 & 4,4 & 209226,86 & 4,0 \\
\hline Total & 5043525,49 & 100,0 & 5230845,13 & 100,0 \\
\hline
\end{tabular}

* FO : Force ouvrière ; CFE-CGC : Confédération française de l'encadrement-Confédération générale des cadres ; CFTC : Confédération française des travailleurs chrétiens ; Unsa : Union nationale des syndicats autonomes.

** Le nombre de voix des syndicats comporte des décimales car les scores obtenus résultent parfois de listes communes, ce qui implique une division des suffrages entre alliés.

Note : les résultats de la CFE-CGC sont donnés sur une base intercatégorielle pour faciliter la comparaison. La loi de 2008 garantit cependant à la CFE-CGC un privilège catégoriel : sa représentativité est calculée à partir des seuls collèges électoraux dans lesquels ses règles statutaires lui donnent vocation à présenter des candidats, c'est-à-dire dans les collèges cadres, agents de maîtrise et assimilés. Dans ce périmètre, son audience est de 18,14\% en 2013 et de 19,39\% en 2017.

Source : données Direction générale du travail (DGT).

présélection par l'État ${ }^{5}$ et la reconnaissance mutuelle ${ }^{6}$. Si l'institution de nouvelles branches dépend toujours d'une procédure volontariste engagée par les organisations professionnelles, le rôle de l'État dans la définition de leur périmètre a en outre été renforcé, suivant les préconisations du rapport Poisson (2009) ${ }^{7}$. De même, en matière de négociation collective, les ordonnances de septembre 2017 réformant le Code du travail consacrent bien la primauté de l'entreprise mais elles reconnaissent un ensemble de thèmes (dits verrouillés ou verrouillables, selon les cas) où continuent de prévaloir les normes négociées dans les branches (minima salariaux, classifications, nombre et durée des contrats à durée déterminée, règles du temps partiel, etc.).

5. On fait référence ici à la présomption irréfragable de représentativité qui était conférée aux cinq confédérations « historiques » CGT, CFDT, CGT-FO, CFTC et CFE-CGC.

6. C'est la signature d'une convention collective qui constitue une branche, et c'est pour signer une convention que se constituent des acteurs, notamment patronaux.

7. Ce rapport, résultant d'une mission confiée en novembre 2008 par le Premier ministre François Fillon au député des Yvelines Jean-Frédéric Poisson, visait à faire le point sur la négociation de branche et sur les obstacles à la réduction du nombre de branches professionnelles. Il préconisait un accompagnement des partenaires sociaux dans le processus de restructuration des branches professionnelles et la définition de critères objectifs permettant d'engager ces restructurations. Ce processus a été défini par les lois du 5 mars 2014, du 17 août 2015, du 8 août 2016 et par l'ordonnance du 22 septembre 2017. 
L'audience électorale de branche, comme celles mesurées au niveau des établissements, des entreprises et sur le plan national interprofessionnel, détermine quels syndicats peuvent prendre part à la négociation collective, signer des accords ou s'y opposer. Ce chiffre objectivant la représentativité syndicale à l'échelle conventionnelle est une nouveauté de la réforme de 2008. Sa production constitue la partie la plus complexe du nouveau régime de représentativité syndicale (COMBREXELLE, 2013). En nous concentrant sur ce chiffre intermédiaire, nous souhaitons attirer l'attention sur ce que «mesurent » précisément les résultats de l'audience syndicale. À première vue, ils s'inscrivent dans le sillage des données électorales ayant permis d'étudier l'évolution des audiences syndicales sur plusieurs décennies. Ils introduisent cependant plusieurs ruptures, que nous analysons. Les résultats de l'audience conventionnelle permettent en outre de dresser un état des lieux du paysage syndical dans les branches, d'objectiver les rapports de force et les ressorts du pluralisme syndical à ce niveau. Décomposer les données de la mesure d'audience au niveau des branches professionnelles dresse ainsi un tableau d'ensemble beaucoup plus complexe que la hiérarchie tracée à l'échelle interprofessionnelle.

Pour mener à bien notre analyse, nous commençons par situer Mars au regard des pratiques antérieures de recueil et d'analyse des données des élections professionnelles, pour montrer ce qui change avec le nouveau dispositif de quantification. Nous nous concentrons ensuite sur le niveau des branches professionnelles, car c'est à cette échelle que la spécificité des résultats produits par le dispositif Mars, qui agrège des données électorales hétérogènes, est la plus visible. Nous terminons en décrivant le paysage des syndicats représentatifs dans les branches tel qu'il ressort de la mesure d'audience : le pluralisme y est bien plus riche qu'au niveau interprofessionnel. Les résultats confirment la suprématie du couple CGT-CFDT, mais sans que la CFDT se « détache » véritablement de sa rivale, contrairement à ce que pourrait laisser penser une interprétation hâtive des résultats de la mesure d'audience interprofessionnelle.

\section{La « mesure » de l'audience syndicale : des dispositifs entre rupture et continuité}

Tracer une continuité entre les mouvements électoraux analysés par les chercheurs à partir des seules élections aux comités d'entreprise (CE) et les résultats rendus publics en 2013 et 2017 est tentant. Pourtant, dans la mesure où la réforme de la représentativité a changé la composition du corps électoral ainsi que la fonction des données d'audience, une telle tentative pose problème sur le plan méthodologique.

\section{Une mise en perspective des audiences syndicales}

Les résultats des élections sociales et professionnelles ont depuis longtemps fait l'objet d'analyses sociologiques (ANDOLFATTO, 1992). L'exercice fut inauguré à l'occasion des élections à la Sécurité sociale organisées de 1946 à 1962 (ColLECTIF, 
1953 ; ADAM, 1964) puis brièvement réactivées en 1983. En instituant des élections générales synchronisées sur le plan national, la réforme des prud'hommes de 1979 a eu pour conséquence de faire de ce scrutin, jusqu'à sa disparition, le principal étalon de la représentativité syndicale (SubILEAu, 1999). Mais ce sont surtout les élections aux CE qui ont fourni matière à l'analyse statistique. Ainsi, à partir de 1966, les entreprises organisant des élections professionnelles ont l'obligation d'en transmettre les procès-verbaux à l'inspection du travail (ADAM, 1968). Cette collecte visait la mise en place d'un instrument statistique permettant de produire une connaissance fiable de la réalité des $\mathrm{CE}$ et de la représentativité des organisations syndicales, attente exprimée au milieu des années 1960 aussi bien par les pouvoirs publics que par des chercheurs et certains syndicats (ANDOLFATTO, 1992). Analysés par les services statistiques du ministère du Travail, connus aujourd'hui sous le nom de Direction de l'animation de la recherche, des études et des statistiques (Dares) ${ }^{8}$, ces résultats donnaient lieu à quatre types d'agrégations - à l'échelle nationale, par région, par secteur d'activité et par taille d'entreprise - au sein desquelles étaient distingués les différents collèges. En raison des modalités de remontée, les données, qui n'étaient pas exhaustives et s'avéraient de qualité variable, faisaient l'objet de redressements statistiques. Elles ont en outre été affectées au cours du temps par des changements de périmètre, de modes de collecte ou de classification introduisant des ruptures de séries statistiques.

L'analyse des élections aux CE a mis en avant deux éléments déterminant l'audience syndicale : «l'effet de structure » et «l'effet d'implantation » (AUJARD, VolKOFF, 1986). Le premier renvoie aux caractéristiques socio-économiques des établissements (taille, secteur d'activité, composition socioprofessionnelle de la maind'œuvre) et le second à la capacité différenciée des organisations syndicales à présenter des listes de candidats. Analysant sous ces deux angles les élections de 1945 jusqu'au début des années 1990, Dominique LABBÉ (1994) constate un déclin des syndicats qu'il explique par trois facteurs correspondant aux trois composantes de l'effet de structure : la baisse de la taille des établissements, qui a nui à tous les syndicats, au profit des listes sans étiquette syndicale ; la désindustrialisation et la montée du secteur tertiaire, qui a nui à la CGT et à la Confédération française de l'encadrement-Confédération générale des cadres (CFE-CGC) et profité aux autres syndicats ; la transformation de la morphologie du salariat (montée des cadres et baisse des premiers collèges ouvriers et employés), qui a nui à la CGT et profité à la CFE-CGC. D. LABBÉ pointe l'érosion constante de la CGT, seule organisation subissant les conséquences de l'évolution des trois composantes de l'effet de structure (elle est passée de $80 \%$ des suffrages exprimés en 1946 à 22,5\% au début des années 1990). Cependant, selon l'auteur, le facteur le plus direct du déclin syndical lors de ces élections tient au deuxième effet, à savoir celui des implantations, implantations dont le recul a réduit les chances des salariés de pouvoir voter pour une liste syndicale.

8. Pour une histoire de cette institution, voir Penissat, 2009. 
Ces analyses ont été menées alors que les syndicats étaient au creux de la vague. Des études portant sur la série ultérieure d'élections (1989-2004) notent une remontée de l'implantation et de l'audience syndicales (JACOD, BEn DhaOU, 2008). Elles constatent un redéploiement de la présence syndicale et une hausse du pluralisme dans les établissements. À la fin de la période, les listes syndicales totalisent $80 \%$ des suffrages, inversant la tendance observée entre 1966 et 1990 où la part des «nonsyndiqués » a augmenté de 12 à 26,6 \% des suffrages (ANDOLFATTO, 1992, p. 114). Au cours de cette période, la CGT et la CFDT se retrouvent au coude à coude. À partir du milieu des années 1990, la présence de la CFDT dans les établissements dépasse légèrement celle de la CGT mais son audience électorale reste inférieure. Tandis que les résultats de la CGT se stabilisent au cours de la décennie 1995-2005, la CFDT, après une croissance continue, connaît un effritement à partir de la fin des années 1990. Ce sont les autres syndicats qui progressent alors le plus : la CFE-CGC et la Confédération française des travailleurs chrétiens (CFTC) ainsi que l'Union nationale des syndicats autonomes (Unsa) et Solidaires. Analysant les chiffres Mars de 2013, Jean-Marie PERNOT (2015) souligne la grande stabilité de ces tendances. Mais la comparaison ne se fait pas à données électorales équivalentes ${ }^{9}$ et c'est précisément ce qui change avec la réforme qui doit retenir ici notre attention.

\section{La réforme de 2008 : rupture dans la quantification des audiences syndicales}

Par le recours à des images comme celles du « baromètre » (ANDOLFATTO, 1992 ; LABBÉ, 1994) ou du « thermomètre » (COMBREXELLE, 2013), les résultats des élections professionnelles sont souvent implicitement désignés comme des phénomènes naturels dont les instruments de mesure permettraient de traduire les oscillations. L'institution du dispositif Mars en 2008 a conforté cette idée en reprenant dans son intitulé la notion de mesure, qui semble référer à une métrologie réaliste. Il serait pourtant plus juste de parler d'un dispositif de quantification de l'audience syndicale. La notion de quantification rappelle en effet que les systèmes de production statistique participent à la mise en forme de la réalité autant qu'ils la mesurent (DESROSIÈRES, 2014). Car pour mesurer, il faut commencer par établir des conventions de mesure, qui définissent des règles de mise en équivalence, de codage et de calcul. Les systèmes de recueil et de traitement des données électorales ne sont donc pas de simples dispositifs techniques, ils sont le produit de décisions et d'arrangements sociaux sur ce qui peut être mesuré et comment. À cet égard, la mise en place de Mars acte une triple rupture, qui touche à la fonction des données, à leur chaîne de production et à leur composition.

Depuis la loi du 20 août 2008, la finalité du recueil des données électorales n'est plus statistique (production de connaissances), mais opérationnelle (support aux actions de la puissance publique). L'audience électorale ne fournit plus seulement matière aux analyses des chercheurs et statisticiens. Elle est devenue le principal critère

9. C'est-à-dire en écartant les voix des non-syndiqués des données de la Dares et en écartant celles des salariés des très petites entreprises (TPE) et de l'agriculture des données Mars. 
d'appréciation de la représentativité légale des syndicats, laquelle fonde non seulement leur droit de participer à la négociation collective, mais aussi la validité des accords ainsi conclus. Les suffrages recueillis par les organisations syndicales acquièrent ainsi une fonctionnalité dans la politique publique de promotion du « dialogue social » et ne sont plus la propriété des statisticiens. Auparavant, le travail de redressement mené par ces derniers devait permettre aux données recueillies de s'approcher de la réalité des audiences syndicales en entreprise. Dorénavant, c'est au contraire l'absence de toute altération statistique qui est censée garantir la « vérité des chiffres » et l'effet de vérité qui va avec, en disant qui est représentatif et qui ne l'est pas : « d'une fiabilité statistique - prise dans un intervalle de confiance - il s'agit de passer à une qualification juridique, c'est-à-dire à des données non traitées statistiquement afin que les partenaires sociaux ne puissent contester devant les tribunaux l'établissement des audiences syndicales » (PENISSAT, 2009, p. 338).

Ce changement d'usage a transformé la procédure de saisie et de contrôle des données, instituant la nouvelle chaîne de production de données électorales sur laquelle repose le dispositif Mars. La Dares a demandé à être dessaisie de cette mission d'analyse des résultats des élections professionnelles, devenus sensibles (ibid., pp. 337-339). La saisie et le contrôle des données ont été entièrement externalisés auprès d'un prestataire privé, chargé de la mise en place d'un Centre de traitement des élections professionnelles (CTEP) auquel sont désormais transmis les procès-verbaux d'élections. Ce travail est mené en lien avec les services de la Direction générale du travail (DGT), qui opèrent d'éventuels retours vers les entreprises en cas d'anomalie ${ }^{10}$. Si tout dispositif de quantification résulte d'une construction sociale, Mars a de surcroît acquis une dimension politique qui était absente du système piloté auparavant par la Dares. Les résultats compilés par le CTEP doivent en effet être validés par les partenaires sociaux dans le cadre d'une institution créée à cet effet, le Haut Conseil du dialogue social (HCDS), avant d'être rendus publics ${ }^{11}$. Dans son compte rendu de la réforme « vue de la salle des machines », l'actuel président du HCDS relate divers cas d'arbitrages rendus dans cette instance (COMBREXELLE, 2013). Il écrit par exemple que « certains soutenaient qu'il ne fallait pas prendre en compte les résultats des élections professionnelles au sein [des] entreprises [à statut], dès lors qu'il n'existait pas de convention collective qui leur soit applicable » (ibid., p. 938). En fin de compte,

10. La saisie et la consultation des procès-verbaux d'élections professionnelles sont accessibles en ligne sur le site : https://www.elections-professionnelles.travail.gouv.fr/, consulté le 20 novembre 2018.

11. Le HCDS, institué par décret le 13 novembre 2008, est composé de cinq représentants des confédérations syndicales représentatives de salariés (CGT, CFDT, CGT-FO, CFTC, CFE-CGC), de cinq représentants des organisations représentatives d'employeurs au niveau national (Medef, CPME [Confédération des petites et moyennes entreprises, anciennement CGPME, Confédération générale des petites et moyennes entreprises], U2P [Union des entreprises de proximité, anciennement UPA, Union professionnelle artisanale], UNAPL [Union nationale des professions libérales], FNSEA [Fédération nationale des syndicats d'exploitants agricoles]), de trois représentants du ministère du Travail et de trois personnalités qualifiées proposées par le ministre du Travail. Jusqu'en 2014, le HCDS fut présidé par Yannick Moreau, ancienne présidente de la section sociale du Conseil d'État, nommée au titre des personnalités qualifiées. Depuis, c'est Jean-Denis Combrexelle, conseiller d'État et ancien directeur général du travail, qui remplit cette tâche. La Dares est représentée par l'entremise d'un des trois représentants du ministère. 
ces suffrages n'entrent pas dans la mesure des audiences de branche mais servent à celle de l'audience interprofessionnelle. A contrario, il a été décidé que les suffrages des journalistes seraient comptés deux fois à l'échelle conventionnelle (pour leur convention propre de métier et pour la convention collective des entreprises de presse dont relèvent leurs employeurs), mais une seule fois à l'échelle interprofessionnelle. En ce qu'elles sont à la fois délibérées politiquement dans le cadre du HCDS (plutôt que d'être redressées statistiquement) et supports de politiques publiques (puisqu'elles conditionnent l'accès des syndicats à diverses prérogatives garanties par l'État et leur poids dans la négociation collective), on peut dire des données produites dans le cadre de Mars qu'elles sont des chiffres politiques.

Une rupture est par ailleurs introduite dans la composition des données puisque Mars s'ouvre à de nouvelles données électorales et en exclut d'autres. Le vote devenant le moyen par lequel les salariés définissent quels syndicats peuvent les représenter, il a fallu y associer les salariés privés d'élections professionnelles. Pour ce faire, plusieurs options ont été avancées par les syndicats, comme la mobilisation des résultats aux élections prud'homales qui, à la différence des élections professionnelles, concernent tous les salariés de droit privé, ou encore la mise en place de nouvelles instances représentatives du personnel (IRP) interentreprises et la synchronisation des élections au niveau de chaque branche professionnelle, sur le modèle de la fonction publique (BARNIER, 2008). La solution finalement retenue combine les résultats des élections aux CE ou, à défaut, les résultats des élections des délégués du personnel ou de la délégation unique du personnel (instances appelées à être remplacées par le comité social et économique ou le conseil d'entreprise suite à la réforme du Code du travail), avec deux dispositifs subsidiaires : le vote des salariés agricoles aux chambres départementales d'agriculture et un scrutin ad hoc organisé tous les quatre ans pour les salariés des très petites entreprises (TPE, jusqu'à dix salariés) qui ne sont pas légalement contraintes d'organiser des élections du personnel ${ }^{12}$.

À l'inverse, les listes sans étiquette syndicale, qui ont été au cœur des interrogations et analyses des chercheurs dans les années 1990-2000, ne sont plus comptabilisées, puisque l'audience est calculée à partir des résultats du premier tour qui est réservé aux listes syndicales. De surcroît, alors qu'on compte environ 19 millions de salariés en France dans le secteur privé, le corps électoral retenu pour la mesure d'audience ne comprend qu'un peu plus de 13 millions de salariés. Outre les situations de carence ${ }^{13}$, qui concerneraient la moitié des exclus de la mesure, rappelons que plus de trois établissements de plus de dix salariés sur dix ne se conforment tout simplement pas à l'obligation légale d'organiser des élections professionnelles (Romans, 2018). Plus largement, les salariés privés d'emploi, les précaires qui ne restent pas assez longtemps dans un établissement pour y voter, les travailleurs des plateformes numériques et

12. Cette dernière disposition a été instituée par la loi du 15 octobre 2010. Les salariés des TPE n'élisent pas de représentants mais se contentent de manifester leur préférence pour une organisation (on parle de vote sur sigle).

13. Il y a carence quand sont organisées des élections du personnel mais qu'aucun candidat ne s'est présenté. Dans ce cas, l'employeur doit dresser un procès-verbal de carence. 
d'autres micro-entrepreneurs, juridiquement autonomes mais économiquement dépendants, les fonctionnaires, etc., sont autant de travailleurs que les syndicats ont vocation à représenter et qui ne participent pas à cette « mesure » de la représentativité. Ces points aveugles sont logiques du point de vue de la fonctionnalité politique du nouveau système : Mars a refondu le dispositif de représentativité légale des syndicats, qui faisait l'objet de critiques diverses depuis de nombreuses années, il n'avait pas vocation à produire une photographie de leur représentativité sociale (BÉROUD et al., 2012 ; GIRAUD et al., 2018, p. 77-106). L'existence d'autres dispositifs statistiques, telle l'enquête REPONSE (Relations professionnelles et négociations d'entreprise), est dès lors d'autant plus importante pour objectiver la diversité des formes de participation et de représentation des travailleurs, au-delà du seul paysage syndical représentatif.

Faire le constat que les chiffres produits par Mars sont à la fois socialement et politiquement construits ne revient pas pour autant à dénoncer un «montage politique » qui déformerait la « réalité » du syndicalisme. Il s'agit de souligner que Mars, comme tout dispositif de quantification, ne se contente pas d'enregistrer la réalité qu'il mesure mais contribue à la façonner. Cela est particulièrement visible à l'échelle des branches professionnelles.

\section{La branche professionnelle, un révélateur de la diversité des ressorts de l'audience syndicale}

Si des données chiffrées permettant d'objectiver les audiences syndicales dans les entreprises et au niveau national interprofessionnel existent depuis longtemps grâce aux élections professionnelles et prud'homales, l'institution d'une audience syndicale de branche est une nouveauté introduite par la réforme de 2008. Avant cette date, les analyses électorales menées grâce aux données collectées par la Dares partaient des résultats globaux, qui étaient ventilés ensuite par grands secteurs d'activité. Ces traitements mettaient en lumière les zones de force et d'influence des différentes organisations. Le niveau de précision a changé avec la réforme de la représentativité qui établit l'audience syndicale de branche. Avec le dispositif Mars, les branches ne sont plus seulement un vague arrière-fond sur lequel projeter les résultats électoraux. Le périmètre de chaque branche structure désormais la production même des résultats, par l'agrégation de scrutins de natures diverses. Cette logique induit des configurations électorales très différentes d'une branche à l'autre, qui sont révélatrices de la diversité des ressorts des audiences syndicales.

\section{Les branches selon le dispositif Mars}

Dans le langage de Mars, une branche correspond au périmètre socio-économique délimité par une convention collective, repérable par un identifiant de convention collective (IDCC). Dans les cas les plus simples, ce périmètre recouvre l'ensemble 
des salariés d'un secteur d'activité au plan national (c'est le cas par exemple de la branche des pompes funèbres, IDCC 0759, ou encore de l'industrie chimique, IDCC 0044). Mais il peut aussi être limité à un territoire plus restreint (les stations-service de Guadeloupe, IDCC 1961) ou à une seule catégorie de personnel (les cadres des travaux publics, IDCC 3212), voire combiner plusieurs critères (les employés de la presse quotidienne parisienne, IDCC 0394 ; les ouvriers des entreprises du bâtiment de plus de dix salariés, IDCC 1597). Loin de répondre à une logique prédéfinie, chaque périmètre est le produit des aléas historiques de la négociation et de la configuration d'acteurs ayant institué la branche. Il dépend en premier lieu des stratégies de négociation des organisations patronales (SELLIER, 1984).

À la fin des années 2000, on compte 680 branches professionnelles; seules 280 d'entre elles regroupent plus de 5000 salariés (Poisson, 2009). Nos calculs portent sur un nombre plus réduit de branches : 555 en 2013 et 458 en 2017. Cette baisse du nombre de branches recensées s'explique par le regroupement de certaines conventions collectives et par la disparition d'autres conventions : d'un cycle à l'autre, le nombre de branches ayant fait l'objet d'un arrêté de représentativité est passé de 473 à 411 . On l'a évoqué plus haut, l'objectif de restructurer les branches professionnelles par la fusion de certaines d'entre elles a été fixé par le législateur dans la suite de la réforme de la représentativité syndicale de 2008. Certains acteurs ont engagé des négociations sur la « rationalisation » de leur paysage conventionnel, notamment dans le secteur de la métallurgie qui compte près de 80 conventions collectives territoriales (CHEUVREUX, RAMBERT, 2017).

Le panorama des branches et leur démographie en 2017 restent cependant relativement inchangés par rapport à la précédente mesure de représentativité en 2013. Une quinzaine de branches (soit 3,1\% du nombre total de branches) regroupent à elles seules près de $40 \%$ de l'ensemble des inscrits, ce qui était déjà le cas en 2013 ; 278 branches $(60,7 \%)$ ont moins de 10000 inscrits en 2017 contre 382 quatre ans plus tôt, ce qui représentait alors plus des deux tiers du nombre de branches $(68,8 \%)$. La principale évolution est donc la disparition progressive des branches les plus petites. Au 31 mars 2018, 156 branches du régime général ont fait l'objet d'une restructuration, en raison du faible nombre de salariés qu'elles couvraient ou bien du caractère restreint de leur périmètre géographique ou catégoriel (MINISTÈRE DU TRAVAIL, 2018).

Parallèlement, le nombre de salariés inclus dans la mesure d'audience (dits « inscrits ») a augmenté d'environ 200000 entre les deux cycles, ce qui résulte d'une amélioration du travail de centralisation des procès-verbaux d'élections professionnelles. La base de données sur laquelle s'appuient nos calculs comptabilise cependant près de 800000 inscrits de moins que pour la mesure d'audience interprofessionnelle, puisque les données disponibles à l'échelle conventionnelle concernent les seules branches devant faire l'objet des arrêtés de représentativité. Des branches trop récentes, celles ayant un trop faible nombre de salariés ou une absence de vie conventionnelle, ainsi que diverses branches mono-entreprise ou régulées par statut ne sont pas concernées par ces 
arrêtés. Ainsi, certains secteurs comme la $\mathrm{SNCF}^{14}$ (plus de 160000 salariés), la RATP ${ }^{15}$ (30 000 salariés) ou le CEA ${ }^{16}$ (19 000 salariés) ne sont pas inclus dans nos données. Cet écart n'est cependant pas rédhibitoire puisque notre analyse porte moins sur la représentativité globale des syndicats que sur la « fabrique » de leur représentativité au sein des branches. En outre, on le verra, les tendances qui se dégagent d'une vision d'ensemble de la représentativité de branche seraient plutôt confortées par la prise en compte des secteurs absents de nos données.

\section{La grande diversité des configurations électorales de branche}

L'audience syndicale de branche est calculée en agrégeant, pour chaque IDCC, les données issues des élections professionnelles avec celles du scrutin propre aux salariés des TPE. Les suffrages des salariés dans les chambres d'agriculture sont décomptés comme formant une branche à part. Cette audience conventionnelle est, selon la configuration des branches, éventuellement assortie d'une audience calculée à une échelle plus large. C'est le cas dans le bâtiment, la métallurgie, les assurances et les travaux publics, où existent des conventions catégorielles et/ou territoriales de niveau infranational.

À l'échelle interprofessionnelle, l'hétérogénéité des données électorales agrégées dans la mesure d'audience peut passer d'autant plus inaperçue que les voix recueillies par les organisations syndicales hors des élections professionnelles ont un poids marginal. Bien que les salariés des TPE comptent pour plus d'un quart du salariat, ils représentent en effet moins de $10 \%$ des suffrages exprimés dans la mesure d'audience. Le poids des salariés agricoles est encore plus infime $(2,5 \%$ des inscrits et $1 \%$ des exprimés). À l'échelle des branches en revanche, la situation est bien différente car le poids des TPE varie fortement d'une branche à l'autre. La proportion de salariés de TPE parmi les inscrits à la mesure d'audience varie ainsi de $100 \%$ (notamment pour la branche du particulier employeur, qui est la première branche en termes d'effectifs avec plus de 660000 inscrits, ou pour certaines branches du bâtiment et du commerce) à moins de $1 \%$ (dans les assurances, la banque ou la sidérurgie par exemple). Le poids global des salariés de TPE, pour les branches étudiées, est de 40,6\% tandis que leur poids médian est de $22,25 \%$, mais avec un écart-type important $(34,31)$, ce qui reflète la grande diversité des situations.

En outre, la probabilité que des élections se tiennent au sein des entreprises dépend fortement de la branche étudiée, en raison du nombre et de la taille variables des établissements et des dynamiques socio-économiques propres à chaque secteur. Afin d'objectiver cette diversité des configurations de branche, nous avons apparié les

\footnotetext{
14. Société nationale des chemins de fer français.

15. Régie autonome des transports parisiens.

16. Commissariat à l'énergie atomique et aux énergies alternatives.
} 
résultats électoraux avec les informations issues des fiches statistiques de la Dares ${ }^{17}$, pour 242 branches (66,2 \% du corps électoral) et pour le cycle 2009-2012. En comparant les données Dares et Mars sur ces branches, on note que seuls 63,4\% des salariés des branches étudiées sont « inscrits » et donc appelés à voter. On l'a dit, les salariés d'entreprises de plus de dix salariés sans IRP ou faisant état de carence syndicale sont de fait exclus du corps électoral. Le chiffre que nous obtenons rejoint par ailleurs le taux de salariés couverts par un délégué syndical, évalué à $64 \%$ en 2017 (RomANs, 2018). Selon les branches, cet écart entre salariat et corps électoral est plus ou moins important. Dans près d'un quart des branches, le corps électoral n'atteint pas la moitié des effectifs salariés comptabilisés. C'est par exemple le cas dans la restauration rapide (IDCC 1501) avec 69614 inscrits pour un effectif de 150100 salariés. Une telle différence s'explique par le morcellement de la branche dont le nombre d'entreprises (19 590) est presque aussi important que le nombre d'établissements (21 170), ce qui démultiplie le risque de non-intégration des salariés au corps électoral. À l'opposé, dans un quart des branches, le corps électoral dépasse les trois quarts des effectifs comptabilisés. De manière surprenante, dans quinze branches, le nombre d'inscrits dépasse même l'effectif enregistré par la Dares, comme dans la distribution directe (IDCC 2372), avec 33713 inscrits pour 24500 salariés. Cette situation s'explique ici par le taux de rotation élevé de la main-d'œuvre et sa concentration dans deux entreprises. Cette concentration facilite l'inscription des salariés au corps électoral, mais comme le nombre d'inscrits est arrêté à des moments différents dans chaque entreprise, il dépasse l'effectif en emploi à un instant $t$ dans la branche.

Chaque branche offre donc une configuration singulière, du fait de la combinaison entre sa taille et son périmètre (régional, national ou catégoriel), de l'équilibre entre scrutins dans les TPE et scrutins pour les IRP ainsi qu'entre petits et grands établissements. Les ressorts de la construction de l'audience syndicale conventionnelle varient en fonction de ces équilibres.

Dans les branches industrielles, où dominent les grandes entreprises et où la part des salariés des TPE est infime, la vie électorale est généralement stable et l'audience syndicale se joue dans les entreprises. Par exemple, dans la branche du caoutchouc (IDCC 0045), la moitié des entreprises ayant dressé des procès-verbaux d'élections avec présence syndicale l'ont fait au cours des deux cycles et ces entreprises représentent plus de $90 \%$ des inscrits ${ }^{18}$. A contrario, dans les branches du bâtiment ou du commerce, l'atomisation et la volatilité des entreprises dominent. Par exemple, dans la branche de l'immobilier (IDCC 1527), seul un quart des entreprises ayant organisé des élections professionnelles ont fait remonter des procès-verbaux au cours des deux cycles, et elles représentent moins de $60 \%$ des inscrits de la branche. L'audience électorale dépend dès lors surtout de la capacité des syndicats à suivre ces

17. Fiches statistiques publiées par la Dares pour l'année 2011. En ligne : https://dares.travail-emploi.gouv.fr/daresetudes-et-statistiques/tableaux-de-bord/les-portraits-statistiques-de-branches-professionnelles/les-250-portraits-statistiques-structurels/article/conventions-collectives-de-branche-fiches-statistiques, consulté le 20 novembre 2018.

18. Ce calcul et les suivants ont été réalisés à partir des données mars 2017 et Dares 2014. 
recompositions en étendant leur présence vers de nouvelles entreprises. Là où les PME sont les plus nombreuses, la simple présentation d'une liste aux élections s'avère déterminante, les scrutins dans les petits établissements et les petites entreprises étant particulièrement peu concurrentiels (HAUTE, 2018). Dans d'autres secteurs, comme la distribution directe déjà mentionnée, mener campagne dans la branche se résume à coordonner les élections dans quelques entreprises qui contrôlent le secteur (YON, 2012). L'implantation reste donc décisive dans la production des audiences syndicales mais se décline différemment selon les configurations électorales.

Tandis que la mobilisation des votes des salariés des TPE ne représente pas un enjeu fort dans certaines branches, dans d'autres en revanche, elle est capitale puisqu' elle constitue le principal ressort de l'audience syndicale. Les travaux consacrés aux élections dans les TPE (REY et al., 2014 ; HAUTE, 2017) montrent que la logique y est assez proche de celle des élections prud'homales : il s'agit en effet d'un scrutin national auquel toutes les organisations peuvent librement participer et le vote, organisé à distance, ne se déroule pas dans les entreprises. Le caractère national du vote est par ailleurs d'autant plus affirmé qu'il n'est pas ici question d'élire des représentants mais d'exprimer sa préférence en votant sur un sigle. Comme pour les élections prud'homales (SuBILEAU, 1999), mais de manière plus accusée encore étant donné les caractéristiques sociales des salariés des TPE et l'enjeu du scrutin, les syndicats sont confrontés au risque de faible participation. Dans ce contexte, la renommée des organisations et leurs capacités militantes sont décisives pour mobiliser les salariés. Certaines organisations ont pu cependant contrebalancer leur faiblesse organisationnelle en déployant des stratégies plus sectorielles. L'Unsa a par exemple développé des actions et une communication postale ciblées auprès des assistantes maternelles ou des salariés de la coiffure, deux branches importantes en termes d'effectifs (IDCC 2395 et 2596), avec respectivement 290000 et 90000 salariés, travaillant principalement dans des TPE. L'Unsa a acquis la première place dans la coiffure, avec plus de $32 \%$ des suffrages, et la troisième dans la branche des assistantes maternelles, derrière deux autres syndicats non confédérés.

Cette diversité des configurations électorales laisse donc penser que la branche, par son périmètre et sa structure, agit comme un « contexte » particulier de production des votes professionnels, au sens de la sociologie électorale (AUDEMARD, 2017) : les comportements électoraux des salariés ne dépendent pas uniquement des caractéristiques individuelles de ces derniers mais oscillent aussi en fonction des «groupes de référence » qui eux-mêmes varient selon les branches.

Notre étude de l'audience syndicale de branche à partir du dispositif Mars met en lumière des résultats hétérogènes, tant entre les branches professionnelles qu'à l'intérieur de chacune d'elles. Du fait de la diversité des configurations électorales, les ressorts de la performance électorale des syndicats ne sont pas les mêmes d'une branche à l'autre. Dans les branches où l'audience syndicale résulte avant tout de l'agrégation des résultats obtenus dans les entreprises, l'échelle conventionnelle a en somme peu d'importance par rapport à celle de l'entreprise. Dans d'autres, à l'inverse, 
le poids du vote des salariés des TPE appelle des stratégies électorales conçues à l'échelle de la branche. Enfin, quand ce sont plutôt les PME qui dominent la branche, une stratégie intermédiaire est nécessaire, articulant présence syndicale en entreprise et pilotage du développement syndical depuis la branche. Cette attention aux contextes conventionnels permet ainsi de confirmer l'importance des effets de structure et d'implantation mentionnés plus haut dans la production des audiences syndicales, tout en précisant que ces effets se déclinent et se combinent différemment d'une branche à l'autre. Après avoir étudié les conditions de production de l'audience syndicale, nous pouvons aborder ce que les résultats électoraux donnent à voir au niveau des branches.

\section{Un état du champ de la représentation syndicale dans les arènes conventionnelles}

L'ancien système basé sur la présomption de représentativité permettait aux cinq confédérations historiques de participer aux négociations des conventions collectives sans être nécessairement présentes dans les périmètres visés par les textes. Fonctionnel dans un contexte où l'hostilité à l'hégémonie cégétiste rapprochait les employeurs, les pouvoirs publics et les autres syndicats, ce système a perdu en légitimité depuis les années 1980 avec l'effritement de la CGT, le basculement de la Confédération nationale du patronat français (CNPF, devenue en 1998 Mouvement des entreprises de France, Medef) vers une stratégie faisant primer la négociation d'entreprise (WEBER, 1986), l'apparition de nouvelles organisations syndicales comme l'Unsa et Solidaires, et l'ouverture de possibilités de déroger au principe de faveur. La suppression de la présomption irréfragable de représentativité en 2008 a mis fin à ce système. Désormais, les syndicats doivent leur participation dans les négociations de branche à leurs résultats électoraux dans le secteur concerné. Ils n'en conservent pas moins un pouvoir de représentation qui dépasse leur ancrage social effectif, puisque la régulation de branche concerne précisément toutes les entreprises couvertes par une convention collective, qu'elles disposent ou non d'une présence syndicale.

Pour étudier le poids des organisations syndicales dans les branches, les seuils retenus pour la mesure d'audience doivent être pris en compte. Ainsi, les organisations syndicales ayant recueilli au moins $8 \%$ des suffrages exprimés sont considérées comme représentatives et peuvent participer aux négociations collectives de branche. Ensuite, les seuils de $30 \%$ et de $50 \%$ permettent à une organisation syndicale d'obtenir une majorité «d'engagement » pour signer un accord ou «d'opposition » pour le rejeter. Ces majorités ne sont pas calculées à partir des suffrages exprimés mais à partir du rapport entre les suffrages recueillis par une organisation syndicale représentative et l'ensemble des suffrages s'étant portés sur toutes les organisations représentatives de la branche : si un syndicat obtient $50 \%$ des voix dans une branche mais qu'aucun autre syndicat ne franchit les $8 \%$, son poids relatif sera de $100 \%$. 
Nous avons effectué nos calculs à partir de la base Mars et non à partir des arrêtés établissant les organisations représentatives, dont la publication pour le cycle 20132016 était encore en cours au moment de l'écriture de cet article. En octobre 2018, 411 arrêtés de représentativité avaient été publiés au Journal officiel par vagues depuis juin 2017. Pour nos calculs, nous avons présupposé que tous les syndicats ayant franchi $8 \%$ dans une branche déposent un dossier de demande de reconnaissance de leur représentativité. Si c'est toujours le cas pour les grandes organisations syndicales, cela l'est nettement moins pour de petites organisations ou pour de petites branches. Nous avons également présumé que l'ensemble des branches au sein desquelles ont été comptabilisés des suffrages a fait l'objet d'un arrêté de représentativité. Or, à l'issue de la première mesure d'audience, faute de vie conventionnelle (COMBREXELLE, 2013), 186 branches n'ont pas fait l'objet d'arrêtés de représentativité. Ces branches relèvent généralement de secteurs en déclin et représentent par conséquent une proportion marginale de l'ensemble des suffrages. Tout comme la mesure d'audience interprofessionnelle publiée par la DGT, les chiffres de la représentativité de branche établis par arrêtés décrivent moins des audiences que des rapports de force dans le champ de la représentation syndicale : ils nous informent sur la configuration et le poids des différentes organisations dans les arènes de la négociation collective.

\section{CGT, CFDT et FO, les trois organisations les plus représentatives}

Si l'on observe le premier seuil, celui de la représentativité, c'est la CFDT qui le franchit le plus souvent dans les branches, mais c'est la CGT qui est représentative dans le périmètre le plus large (tableau 2). Les différences sont cependant mineures. En 2017, sur 458 branches, la CGT est absente de 15 branches, qui couvrent un peu plus de 7000 inscrits. Dans les 443 branches où se sont exprimés des suffrages pour la CGT, celle-ci n'atteint pas le seuil des $8 \%$ dans 33 branches couvrant 290063 inscrits. Elle est donc représentative dans 410 branches couvrant un total de 12175038 salariés, soit 97,6 \% de l'ensemble des inscrits. La CFDT est absente dans 9 branches couvrant moins de 4000 inscrits. Elle est donc présente dans les 449 autres, mais n'atteint pas le seuil de $8 \%$ dans 31 branches, qui représentent 413941 inscrits. Elle est représentative dans 418 branches couvrant 12054686 inscrits, soit 96,6\% des inscrits. D'un cycle à l'autre, on constate que le champ de représentativité des deux principales confédérations est quasiment identique, alors que la CFDT était légèrement en tête à l'issue du cycle de 2013. Seule Force ouvrière (FO) peut prétendre rivaliser avec les deux principales confédérations en termes de représentativité de branche. Elle n'est absente que dans 19 branches couvrant un peu plus de 6000 inscrits et dépasse le seuil de représentativité dans 368 des 439 autres branches, soit un périmètre de 11576803 inscrits (92,8\% du total).

Le « champ de représentativité » des autres organisations syndicales est nettement plus réduit. La CFTC « rayonne » sur un peu plus de la moitié du corps électoral total. Mais la légère progression de son champ de représentativité dans les branches masque 
un changement majeur intervenu avec la deuxième mesure d'audience. La publication des résultats du deuxième cycle en 2017 marque en effet la fin des dispositions transitoires qui permettaient aux organisations représentatives avant 2008 de siéger dans toutes les branches, même lorsqu'elles n'atteignaient pas le seuil des $8 \%$. Dès lors, la hausse légère de la CFTC est à relativiser car cette organisation va en même temps perdre sa représentativité de branche pour près de la moitié des salariés.

La situation est différente pour la CFE-CGC et mérite quelques explications. Les données concernant cette organisation dans le tableau 2 sont calculées selon une convention particulière, qui renvoie au « privilège catégoriel » déjà évoqué plus haut. La CFE-CGC, qui se présente quasi exclusivement dans les collèges dédiés aux cadres et aux techniciens et agents de maîtrise, voit sa représentativité calculée sur la base de ses résultats dans ces seuls collèges, sans être pour autant exclue des négociations qui porteraient sur les autres catégories de personnel. A contrario, les autres organisations voient leur représentativité systématiquement calculée sur une base intercatégorielle, bien qu'elles ne soient pas toujours présentes chez les cadres et agents de maîtrise (ce qui reste souvent le cas des listes CGT, par exemple). C'est pourquoi on parle $\mathrm{du}$ « privilège catégoriel » de la CFE-CGC. Grâce à cette règle, la CFE-CGC est représentative pour le cycle 2013-2016 dans près de 300 branches couvrant 9,8 millions de salariés, soit 78,6\% du corps électoral total, ce qui la rapproche des trois organisations syndicales les plus représentatives que sont la CFDT, la CGT et FO. On mesure mieux l'impact de ce privilège catégoriel en comparant les résultats obtenus selon cette convention à ceux que donnerait une mesure sur une base intercatégorielle. En changeant de règle de calcul, la CFE-CGC ne serait plus représentative, pour le cycle 2013-2016, que dans 164 branches couvrant 5175185 salariés, soit 41,5\% du corps électoral total. Le privilège catégoriel permet donc à la CFE-CGC de quasiment doubler son champ de représentativité.

TABLEAU 2 - Champ de représentativité selon l'organisation syndicale (2013 et 2017)

\begin{tabular}{lccc|ccc}
\hline $\begin{array}{l}\text { Organisations } \\
\text { syndicales }\end{array}$ & $\begin{array}{c}\text { Nombre de } \\
\text { branches } \\
\text { où chaque } \\
\text { syndicat est } \\
\text { représentatif }\end{array}$ & $\begin{array}{c}\text { Nombre de } \\
\text { salariés } \\
\text { inscrits } \\
\text { couverts par } \\
\text { ces branches }\end{array}$ & $\begin{array}{c}\text { \% de salariés } \\
\text { inscrits } \\
\text { couverts par } \\
\text { ces branches }\end{array}$ & $\begin{array}{c}\text { Nombre de } \\
\text { branches } \\
\text { où chaque } \\
\text { syndicat est } \\
\text { représentatif* }\end{array}$ & $\begin{array}{c}\text { Nombre de } \\
\text { salariés } \\
\text { inscrits } \\
\text { couverts par } \\
\text { ces branches }\end{array}$ & $\begin{array}{c}\text { \%de salariés } \\
\text { inscrits } \\
\text { couverts par } \\
\text { ces branches }\end{array}$ \\
\hline CFDT & 492 & 12148577 & 95,6 & 418 & 12054686 & 96,7 \\
CGT & 482 & 11992918 & 94,4 & 410 & 12175038 & 97,7 \\
FO & 434 & 11823967 & 93,1 & 349 & 11570561 & 92,8 \\
CFTC & 229 & 6414758 & 50,5 & 203 & 6713110 & 53,9 \\
CFE-CGC & 332 & 9587646 & 78,3 & 302 & 9798279 & 78,6 \\
Unsa & 82 & 2320852 & 18,3 & 86 & 3388430 & 27,2 \\
Solidaires & 41 & 593653 & 4,7 & 35 & 750382 & 6,0 \\
\hline
\end{tabular}

* Il y a moins de branches en 2017 (458) qu'en 2013 (555).

Source : données DGT. Traitements des auteurs. 
Pour les organisations non représentatives, le champ de représentativité est bien plus étroit : l'Unsa est représentative dans moins d'une centaine de branches regroupant 3,4 millions de salariés et Solidaires dans 35 branches couvrant moins d'un million d'inscrits.

Ces évolutions sont à mettre en rapport avec l'évolution globale des résultats électoraux de ces organisations. Ainsi, dans son champ de représentativité, la progression de l'Unsa est nette : si elle ne gagne que peu de branches, celles-ci couvrent un million de salariés en plus. Compte tenu de son privilège catégoriel, la progression de la CFE-CGC est moins visible.

\section{Les formes plurielles du pluralisme syndical}

Au-delà des cinq confédérations jusqu'alors présumées représentatives et des outsiders reconnus dans le privé que sont l'Unsa et Solidaires, on constate une plus grande diversité d'acteurs dans les branches professionnelles. Trois logiques d'ordre politique, territorial et corporatif sont au principe de ce pluralisme syndical.

Sur le plan national interprofessionnel, ce sont prioritairement des divergences d'ordre politique qui fondent l'existence d'autres organisations prétendant à la représentation des salariés, comme la Confédération autonome du travail (CAT), désignée par ses concurrents comme un syndicat « jaune » (c'est-à-dire soupçonné d'être de connivence avec le patronat), et les Confédérations nationales du travail (CNT et CNTSolidarité ouvrière), de sensibilité anarcho-syndicaliste. Ces organisations n'atteignent presque jamais le seuil de la représentativité de branche. C'est le vote national dans les TPE qui leur permet d'acquérir une visibilité nationale, mais leur implantation est très localisée et correspond à des logiques d'entreprise ou de bassin d'emploi.

Il existe aussi une réalité interprofessionnelle limitée à certains territoires, de syndicats régionalistes et ultramarins. Du côté régionaliste, seul le Syndicat des travailleurs corses franchit les $8 \%$ dans quelques branches. En Guadeloupe, Martinique ou Guyane, les syndicats ultramarins dominent souvent les scènes paritaires des branches circonscrites à leur territoire. Les arrêtés de représentativité dans les branches de 2013 montrent que les organisations métropolitaines ne sollicitent même pas la représentativité dans ces arènes ultramarines, alors qu'elles le font quasi systématiquement pour les branches métropolitaines.

Au total, une quarantaine d'organisations syndicales ont dépassé le seuil de $8 \%$ dans au moins une branche professionnelle. Le principal facteur de pluralisme syndical relève ainsi de logiques corporatives qui se traduisent par l'existence de syndicats autonomes inscrits dans des métiers et des secteurs d'activité particuliers. Ce phénomène doit cependant être pondéré par le fait que la plupart des branches ont un périmètre réduit. Hormis deux syndicats autonomes dans la branche des assistantes maternelles du particulier employeur (286 000 inscrits) et le Syndicat professionnel de l'enseignement libre catholique (SPELC) dans huit conventions de l'enseignement privé couvrant environ 120000 salariés, aucune organisation n'est représentative au 
sein d'une branche de plus de 100000 salariés. Quelques syndicats autonomes sont représentatifs dans des branches couvrant plusieurs dizaines de milliers de salariés, notamment à Pôle emploi, dans les branches de l'audiovisuel, ou encore chez les gardiens d'immeubles.

\section{Un poids dans la négociation qui distingue la CGT et la CFDT}

Si l'on s'intéresse aux deux autres seuils indiqués plus haut qui permettent à une organisation syndicale de disposer d'une majorité «d'engagement» ou « de blocage » dans la négociation, ce sont de nouveau la CGT et la CFDT qui se détachent, y compris de FO. La CGT dispose de majorités absolues (50\% ou plus) dans 49 branches représentant 331000 salariés, notamment celles délimitées par certaines conventions collectives de la presse et de la métallurgie. À l'inverse, la CFDT ne détient la majorité absolue que dans 37 branches représentant 120000 salariés. Ces situations d'hégémonie syndicale sont rarissimes : elles ne correspondent qu'à $2,75 \%$ du champ de représentativité de la CGT et qu'à $1 \%$ de celui de la CFDT.

Le seuil des $30 \%$, permettant de signer seul un accord, est plus intéressant car les organisations le franchissent plus souvent. Ainsi la CGT a un poids supérieur ou égal à $30 \%$ dans 215 branches représentant 5,9 millions de salariés, soit quasiment la moitié de son champ de représentativité, contre 197 branches et 4,6 millions pour la CFDT (environ $38 \%$ de son champ de représentativité). Certes, la CFDT obtient un résultat électoral global supérieur à la CGT et ses résultats sectoriels sont plus homogènes que ceux de la CGT. En outre, la CGT recule fortement, y compris dans ses bastions. Elle reste cependant plus souvent un acteur clé dans la négociation collective de branche, et demeure plus souvent capable d'engager seule sa signature. Ce constat doit cependant être tempéré par le fait que la CGT est l'organisation qui use le moins souvent de cette prérogative, puisque son taux de signature oscille entre 29 et $40 \%$ depuis le milieu des années 2000, tandis que les autres confédérations représentatives signent entre deux tiers et trois quarts des accords interprofessionnels et de branche (Ministère du TraVAiL, 2018).

Le seuil des $30 \%$ est rarement atteint par les autres centrales syndicales, y compris par FO qui ne le franchit que dans 35 branches représentant à peine 550000 salariés (soit moins de $5 \%$ de son champ de représentativité). C'est le cas notamment dans la branche des organismes de sécurité sociale, de plusieurs branches de la métallurgie et du bâtiment ou encore de branches du secteur de la restauration et du tourisme. La CFE-CGC atteint un poids égal ou supérieur à $30 \%$ dans 14 branches regroupant près de 710000 inscrits, par exemple dans la branche des ingénieurs et cadres de la métallurgie (350 000 inscrits) et dans celle de la banque (220 000 inscrits). La CFTC dispose de majorités d'engagement dans 21 branches comptant un peu moins de 300000 salariés, en particulier dans l'enseignement privé et le commerce (chaussures, articles de sport, électroménager, etc.). L'Unsa et Solidaires ont un poids supérieur ou égal à $30 \%$ dans respectivement sept et cinq branches représentant 134000 et 
36000 salariés. Dans le cas de l'Unsa, ces branches sont diverses, incluant notamment la convention collective de la coiffure (près de 90000 inscrits) et la branche des agents des Caisses d'épargne (40 000 inscrits). Pour Solidaires, il s'agit principalement des branches de la presse, notamment de la convention collective des journalistes.

Ces données nous permettent de saisir l'évolution du poids relatif des différents acteurs syndicaux dans la négociation collective, avec une limite toutefois. Les majorités d'engagement ou de refus d'un accord collectif à l'échelle d'une branche ne reposent en effet que très rarement sur une seule organisation. Ce sont souvent des coalitions d'acteurs qui signent ou rejettent les accords. Par exemple, à Pôle emploi, le recul de la CGT a entraîné la perte de la majorité absolue pour la coalition CGT, FO et Fédération syndicale unitaire (FSU), qui défendait jusqu'alors des positions convergentes, ce qui rebat les cartes dans la négociation collective au sein de la branche. Selon les branches, ces coalitions n'ont ni le même périmètre, ni la même stabilité. Les situations sont dès lors trop variables pour qu'un traitement statistique permette de saisir des effets directs de l'évolution des rapports de forces syndicaux sur la négociation collective.

L'entrée par les chiffres de la représentativité de branche permet de relativiser les commentaires mettant l'accent sur le déclin de la CGT et la position de première organisation conquise par la CFDT dans le secteur privé. En matière de poids dans la négociation collective, le constat est en effet plus nuancé. La CGT et la CFDT, mais aussi FO, ont à peu près les mêmes capacités d'accès aux arènes conventionnelles, franchissant presque toujours le seuil des $8 \%$. L'impact du «privilège catégoriel » permet, quant à lui, à la CFE-CGC de limiter son « décrochage » vis-à-vis de ces trois confédérations. Le pluralisme est certes bien plus large dans les branches qu'à l'échelle interprofessionnelle, mais les acteurs syndicaux invisibles au niveau national interprofessionnel et qui comptent à l'échelle professionnelle sont peu nombreux. Si l'on s'intéresse aux seuils de $30 \%$ (signature d'un accord) et de $50 \%$ (droit d'opposition), seules la CGT et la CFDT se détachent. Cependant, le pouvoir de signer seul un accord reste plus souvent entre les mains de la CGT que de la CFDT. Quant au pouvoir de blocage, s'il est plus souvent détenu par la CGT, il reste très rare au niveau conventionnel. De tels résultats décrivent un paysage conventionnel avant tout structuré par le duopole CGT-CFDT. Des recherches plus poussées sur les logiques d'alliance seraient cependant nécessaires pour améliorer notre connaissance des dynamiques de la négociation collective au sein des branches. Si l'entrée par les branches ne donne certes à voir qu' une image très imparfaite du paysage syndical concret ou des rapports entre salariés et syndicats, elle permet toutefois de mieux souligner les capacités d'action différenciées des syndicats, en termes d'accès et de poids dans la négociation collective mais aussi de ressources militantes et financières. 
Cet article a cherché à montrer l'intérêt d'étudier la représentativité syndicale à l'échelle des branches et en leur sein. L'analyse des chiffres de la mesure d'audience conventionnelle met en lumière la rupture avec l'ancien dispositif statistique géré par la Dares et le caractère hybride du nouveau dispositif de quantification, Mars, qui produit en effet deux types d'information.

En premier lieu, mais à la condition de désagréger les données statistiques produites dans le cadre des branches, les résultats de la représentativité syndicale permettent, dans le sillage des analyses électorales antérieures, de prolonger la connaissance des déterminants de l'audience syndicale. La combinaison des données issues des élections professionnelles et du scrutin organisé pour les salariés des TPE dessine des configurations électorales variables d'une branche à l'autre. Comprendre la production des audiences syndicales à l'échelle des branches suppose de tenir compte de ces configurations qui modulent l'impact respectif des effets de structure et d'implantation dans les performances électorales des syndicats.

Dans la logique du nouveau dispositif, les résultats de l'audience syndicale servent à la détermination des acteurs syndicaux représentatifs. C'est le deuxième niveau d'information que produit Mars, celui des chiffres « politiques ». Les étudier permet de dresser un état des lieux du paysage syndical représentatif au sein des arènes paritaires de branche. En donnant à voir un paysage qui ne se résume ni au simple schéma de cinq confédérations représentatives plus deux ou trois outsiders, ni au morcellement des entreprises, les branches offrent une échelle d'observation intermédiaire grâce à laquelle il est possible de déceler et d'expliquer le poids des différentes organisations syndicales dans la négociation. Si les fédérations affiliées aux trois confédérations CGT, CFDT et FO se démarquent des autres par leur capacité à parler au nom des salariés du secteur marchand et associatif dans leur diversité, seules les fédérations affiliées à la CGT et à la CFDT peuvent réellement jouer un rôle clé dans les négociations conventionnelles. C'est en effet autour d'elles qu'il est le plus souvent possible de construire des majorités de négociation ou de blocage. Malgré son passage en première position dans la mesure d'audience interprofessionnelle, la CFDT reste derrière la CGT de ce point de vue. Ce sont encore les fédérations affiliées à cette dernière qui détiennent plus souvent une position de pivot dans la négociation conventionnelle. Ce constat ne présume cependant pas des dynamiques effectives de la négociation de branche, qui dépendent aussi des stratégies de coalition entre acteurs syndicaux.

La primauté maintenant donnée aux accords d'entreprise dans la hiérarchie des normes produites par la négociation collective a réduit le rôle de la branche dans l'égalisation des conditions de la concurrence économique et sociale entre entreprises et salariés au sein d'un même secteur d'activité. La norme conventionnelle conserve cependant une fonction cruciale d'encadrement des relations de travail dans les petites entreprises où le «dialogue social » viendrait à manquer : depuis la loi Travail de 2016, les employeurs d'entreprises de moins de 50 salariés ont ainsi la possibilité de se saisir d'accords-types élaborés au niveau des branches. Elle conserve en outre un rôle de verrou sur divers points tels que les minima salariaux et les classifications, 
le nombre et la durée des contrats à durée déterminée, les règles du temps partiel. La conquête de la représentativité la plus large et la plus incontestable à l'échelle conventionnelle reste donc un enjeu de première importance pour les organisations syndicales. Pour ce faire, ces dernières ont besoin de s'appuyer sur une connaissance fine de l'audience syndicale, qui implique à la fois une analyse électorale multi-niveaux (établissements, entreprises, branches et interprofession) et une prise en compte des spécificités de chaque configuration de branche, des jeux d'acteurs et des caractéristiques socio-économiques des salariés et des entreprises. L'étude circonstanciée des contextes conventionnels s'avère d'autant plus importante que le paysage des branches est appelé à évoluer. Dans la perspective d'une réduction considérable du nombre des branches, les rapports de force y existant seront à la fois un déterminant et un produit des négociations visant à définir les nouveaux périmètres conventionnels et les garanties en leur sein.

\section{BIBLIOGRAPHIE}

AdAm G. (1964), Atlas des élections sociales en France, Paris, Armand Colin.

ADAm G. (1968), «La représentativité des organisations syndicales. Enquête sur les élections professionnelles », Revue française de science politique, vol. 18, $\mathrm{n}^{\circ} 2$, pp. 278-314.

Andolfatto D. (1992), L'Univers des élections professionnelles. Travail et société au crible des urnes, Paris, Éditions ouvrières.

Andolfatto D. (2018), Que pèsent les syndicats ?, Note, Paris, Fondation pour l'innovation politique.

Audemard J. (2017), « De quoi le contexte est-il le nom ? Critique de l'usage de la notion de contexte en sociologie électorale », Revue française de science politique, vol. $67, \mathrm{n}^{\circ} 2$, pp. 271-289.

Aujard J.-P., VolkofF S. (1986), « Une analyse chiffrée des audiences syndicales », Travail et Emploi, $\mathrm{n}^{\circ} 30$, pp. 47-57.

BARNIER L.-M. (coord.) (2008), Revendiquer et s'organiser! Représentativité syndicale et démocratie sociale, Paris, Fondation Copernic, Syllepse.

BÉroud S., Le CROM J.-P., YoN K., (2012), « Représentativités syndicales, représentativités patronales. Règles juridiques et pratiques sociales. Introduction », Travail et Emploi, ${ }^{\circ} 131$, pp. 5-22.

BÉROUD S., YON K. (2014), « Représenter les salariés dans l'entreprise après la loi du 20 août 2008 : sur les limites de la "démocratie sociale" », Politiques de communication, n ${ }^{\circ}$ 2, pp. 51-78.

CheuvreuX M., RAMBERT L. (2017), « Dialogue social sectoriel et décentralisation des négociations : étude comparée France/Allemagne », Documents de travail de la DG Trésor, n $^{\circ}$ 2017/01.

Collectif (1953), «Les élections sociales comparées aux élections politiques », Revue française de science politique, vol. $3, \mathrm{n}^{\circ} 2$. 
CombreXelle J.-D. (2013), « La réforme de la représentativité syndicale : vue de la salle des machines », Droit social, $\mathrm{n}^{\circ}$ 11, pp. 932-940.

DesRosières A. (2014), Prouver et gouverner. Une analyse politique des statistiques publiques, Paris, La Découverte.

GIRAud B., Yon K., BÉROud S. (2018), Sociologie politique du syndicalisme, Malakoff, Armand Colin.

Haute T. (2016), « Participer ou non aux scrutins professionnels : quelles inégalités ? », in Fretel A., Bory A., Célérier S., Jany-Catrice F., Politiques sociales en mutation : quelles opportunités et quels risques pour l'État social ?, XXXVII Journées de l'Association d'économie sociale, coll. « Cahiers du Cirtes », Hors-série $n^{\circ}$ 6, Louvain-la-Neuve, Presses universitaires de Louvain, pp. 445-462.

HAUTE T. (2017), « Des votes inexplicables ? Une analyse électorale des scrutins TPE », communication au Congrès de l'Association française de sociologie, Amiens.

HAUte T. (2018), «Évolutions du paysage syndical et évolutions du salariat : une analyse des élections aux comités d'entreprise (2009-2016) », La revue de l'Ires, $\mathrm{n}^{\circ}$ 94-95, pp. 57-89.

Hege A., Dufour C., Kahmann M. (2015), « La loi du 20 août 2008 et les fondements de la représentation syndicale », La revue de l'Ires, n 87, pp. 95-123.

JACOD O., Ben DhaOu R. (2008), «Les élections aux comités d'entreprise de 1989 à 2004 : une étude de l'évolution des implantations et des audiences syndicales », Document d'études, $\mathrm{n}^{\mathrm{o}} 137$, Dares.

LABBÉ D. (1994), Les Élections aux comités d'entreprise (1945-1993), Saint-Martin-d'Hères, Cerat.

Le Digol C., Voilliot C. (2011), « Hors champ. L'analyse politique et les élections professionnelles », in Leclerc O., Lyon-Caen A. (dir.), L'Essor du vote dans les relations professionnelles. Actualités françaises et expériences européennes, Paris, Dalloz, pp. 41-51.

Ministère du Travail (2018), La Négociation collective en 2017. Édition 2018, coll. « Bilans $\&$ rapports », Paris, Ministère du Travail.

Penissat É. (2009), L'État des chiffres. Sociologie du service de statistique et des statisticiens du ministère du Travail et de l'Emploi (1945-2008), thèse de doctorat en sociologie, Paris, École des hautes études en sciences sociales.

Pernot J.-M. (2015), « Le nouvel artefact de la représentativité », texte inédit.

PoISSON J.-F. (2009), Rapport sur la négociation collective et les branches professionnelles. Rapport au Premier ministre, coll. « Collection des rapports officiels », Paris, La Documentation française.

Rey F. (coord.), Borisova K., ThoboIs P. (2014), Construire un syndicalisme de proximité pour les petites entreprises. Stratégies, pratiques et actions syndicales en direction des salariés des petites entreprises : le cas de la CFDT, Rapport de recherche, Lise (Cnam), CFDT.

Romans F. (2018), « Les relations professionnelles en 2017 : un panorama contrasté du dialogue social dans les établissements ? », Dares analyses, $\mathrm{n}^{\circ} 015$. 
SELLIER F. (1984), La Confrontation sociale en France, 1936-1981, Paris, Presses universitaires de France.

Subileau F. (1999), « Les élections prud'homales. Participation, représentativité, légitimité », Politix, $\mathrm{n}^{\circ}$ 47, pp. 151-166.

TAllard M. (2004), Action publique et régulation de branche de la relation salariale, Paris, L'Harmattan.

Weber H. (1986), Le Parti des patrons. Le CNPF (1946-1986), Paris, Seuil.

Yon K. (2012), « Représentation du travail et représentativité syndicale aux marges du salariat : le cas de la distribution directe », Travail et Emploi, $\mathrm{n}^{\circ}$ 131, pp. 103-118. 
